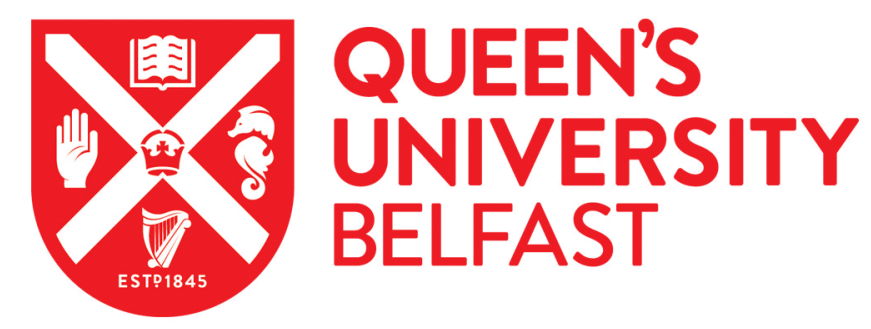

\title{
Exploring variations in ovarian cancer survival by age and stage (ICBP SurvMark-2) : A population-based study
}

Cabasag, C. J., Butler, J., Arnold, M., Rutherford, M., Bardot, A., Ferlay, J., Morgan, E., Møller, B., Gavin, A., Norell, C. H., Harrison, S., Saint-Jacques, N., Eden, M., Rous, B., Nordin, A., Hanna, L., Kwon, J., Cohen, P. A., Altman, A. D., ... Soerjomataram, I. (2020). Exploring variations in ovarian cancer survival by age and stage (ICBP SurvMark-2) : A population-based study. Gynecologic Oncology.

https://doi.org/10.1016/j.ygyno.2019.12.047

Published in:

Gynecologic Oncology

Document Version:

Peer reviewed version

Queen's University Belfast - Research Portal:

Link to publication record in Queen's University Belfast Research Portal

\section{Publisher rights}

Copyright 2020 Elsevier

This manuscript is distributed under a Creative Commons Attribution-NonCommercial-NoDerivs License

(https://creativecommons.org/licenses/by-nc-nd/4.0/), which permits distribution and reproduction for non-commercial purposes, provided the author and source are cited.

\section{General rights}

Copyright for the publications made accessible via the Queen's University Belfast Research Portal is retained by the author(s) and / or other copyright owners and it is a condition of accessing these publications that users recognise and abide by the legal requirements associated with these rights.

Take down policy

The Research Portal is Queen's institutional repository that provides access to Queen's research output. Every effort has been made to ensure that content in the Research Portal does not infringe any person's rights, or applicable UK laws. If you discover content in the Research Portal that you believe breaches copyright or violates any law, please contact openaccess@qub.ac.uk. 
1 Exploring variations in ovarian cancer survival by age and stage (ICBP SurvMark-2): a

2 population-based study

3

4 Citadel J Cabasag $^{\mathrm{a}}$, John Butler ${ }^{\mathrm{b}}$, Melina Arnold ${ }^{1}$, Mark Rutherford ${ }^{\mathrm{a}, \mathrm{c}}$, Aude Bardot ${ }^{\mathrm{a}}$, Jacques Ferlay,

5 Eileen Morgan ${ }^{\mathrm{d}}$, Bjørn Møller ${ }^{\mathrm{e}}$, Anna Gavin ${ }^{\mathrm{d}}$, Charles Norell ${ }^{\mathrm{f}}$, Samantha Harrison ${ }^{\mathrm{f}}$, Nathalie Saint-

6 Jacques $^{\mathrm{g}}$, Michael Eden $^{\mathrm{h}}$, Brian Rous $^{\mathrm{h}}$, Andy Nordin ${ }^{\mathrm{i}}$, Louise Hanna $^{\mathrm{j}}$, Janice Kwon ${ }^{\mathrm{k}}$, Paul Cohen $^{1, \mathrm{~m}}$,

7 Alon Altman ${ }^{\mathrm{n}}$, Lorraine Shack ${ }^{\mathrm{o}}$, Serena Kozie ${ }^{\mathrm{p}}$, Gerda Engholm ${ }^{\mathrm{q}}$, Prithwish De ${ }^{\mathrm{r}}$, Peter Sykes ${ }^{\mathrm{s}}$, Geoff

8 Porter $^{\mathrm{t}}$, Sarah Ferguson ${ }^{\mathrm{u}}$, Paul Walsh ${ }^{\mathrm{v}}$, Richard Trevithick ${ }^{\mathrm{w}}$, Dianne O’Connell ${ }^{\mathrm{x}}$, Freddie Bray ${ }^{\mathrm{a}}$,

$9 \quad$ Isabelle Soerjomataram ${ }^{\mathrm{a}}$

10

${ }^{a}$ Section of Cancer Surveillance, International Agency for Research on Cancer (IARC/WHO), 150

Cours Albert Thomas, Lyon, 69372 CEDEX 08, France

$13 \quad{ }^{\mathrm{b}}$ Royal Marsden Hospital, Fulham Road, London SW3 6JJ, England, UK

$14{ }^{\mathrm{c}}$ Biostatistics Research Group, Department of Health Sciences, University of Leicester, University

15 Road, Leicester, LE1 7RH, UK

d Northern Ireland Cancer Registry, Centre for Public Health, Queen's University Belfast, Belfast,

17 Northern Ireland, UK

${ }^{\mathrm{e}}$ Cancer Registry of Norway, Institute of Population-based Cancer Research, P.O. Box 5313

Majorstuen, 0304 Oslo, Norway

${ }^{\mathrm{f}}$ Policy \& Information Directorate, Cancer Research UK, London, England, UK

${ }^{g}$ Nova Scotia Health Authority Cancer Care Program, Registry \& Analytics, 1276 South Street,

Halifax, B3H 2Y9, NS, Canada

${ }^{\mathrm{h}}$ National Cancer Registrations and Analysis Service, Public Health England, Wellington House,

${ }^{\text {i }}$ East Kent Hospitals University National Health Service Foundation Trust, Kent, England, UK

${ }^{j}$ Velindre National Health Service Trust, Cardiff, England, UK

$27{ }^{\mathrm{k}}$ Department of Obstetrics and Gynecology, Division of Surgical Oncology, University of British

28 Columbia, and British Columbia Cancer Agency, Vancouver, British Columbia, Canada 
${ }^{1}$ University of Western Australia, Division of Obstetrics and Gynaecology, Crawley, Western

Australia, Australia

${ }^{\mathrm{m}}$ St John of God Subiaco Hospital, Subiaco, Western Australia, Australia

${ }^{\mathrm{n}}$ Department of Obstetrics, Gynecology and Reproductive Sciences, University of Manitoba,

Winnipeg, MB R3A 1R9, Canada

${ }^{\circ}$ Cancer Control Alberta, Alberta Health Services, 2210 2nd Street, SW Calgary AB, T2S 3C3,

Canada

$36 \quad{ }^{\mathrm{p}}$ Registry Department, Saskatchewan Cancer Agency, Regina, SK S4W 0G3 Canada

$37 \quad{ }^{\mathrm{q}}$ Danish Cancer Society, Strandboulevarden 49, 2100 Copenhagen, Denmark

${ }^{\mathrm{r}}$ Surveillance \& Cancer Registry, Cancer Care Ontario, 620 University Ave., Toronto, ON M5G 2L7,

39 Canada

$40 \quad{ }^{\mathrm{s}}$ University of Otago, Christchurch, New Zealand

$41{ }^{\mathrm{t}}$ Canadian Partnership Against Cancer, 145 King Street West, Suite 900, Toronto, Ontario M5H 1J8,

42 Canada

$43{ }^{\mathrm{u}}$ Gynecologic Oncology, Princess Margaret Hospital Cancer Centre, Toronto, Ontario, Canada

$44 \quad{ }^{v}$ National Cancer Registry Ireland, Cork Airport Business Park, Kinsale Road, Cork T12 CDF7,

45 Ireland

$46{ }^{\mathrm{w}}$ Western Australia Cancer and Palliative Care Network Policy Unit, Health Networks Branch,

47 Department of Health, Perth, Western Australia, Australia

$48{ }^{\mathrm{x}}$ Cancer Research Division, Cancer Council New South Wales, Sydney, New South Wales, Australia

\section{Correspondence to:}

52 Citadel J. Cabasag, PhD, MS

53 Section of Cancer Surveillance

$54 \quad$ International Agency for Research on Cancer

55150 cours Albert Thomas

$56 \quad 69372$ Lyon CEDEX 08, France 
57 Email: CabasagC@,fellows.iarc.fr

58 Telephone: +33472738015

59 Fax: +33472738696

60

61

62

63

64

65

66

67

68

69

70

71

72

73

74

75

76

77

78

79

80

81

82

83

84 


\section{Abstract}

Objective: The study aims to evaluate the differences in ovarian cancer survival by age and stage at

Methods: We analyzed data from 58,161 women diagnosed with ovarian cancer during 2010-2014, followed until 31 December 2015, from 21 population-based cancer registries in Australia, Canada, Denmark, Ireland, New Zealand, Norway, and United Kingdom. Comparisons of 1-year and 3-year age- and stage-specific net survival (NS) between countries were performed using the period analysis approach.

Results: Minor variation in the stage distribution was observed between countries, with most women being diagnosed with 'distant' stage (ranging between $64 \%$ in Canada and $71 \%$ in Norway). The $3-$ year all-ages NS ranged from 45-57\% with Australia (56\%) and Norway (57\%) demonstrating the highest survival. The proportion of women with 'distant' stage was highest for those aged 65-74 and 75-99 years and varied markedly between countries (range:72-80\% and $77-87 \%$, respectively). The oldest age group had the lowest 3-year age-specific survival (20-34\%), and women aged 65-74 exhibited the widest variation across countries (3-year NS range: 40-60\%). Differences in survival between countries were particularly stark for the oldest age group with 'distant' stage (3-year NS range: $12 \%$ in Ireland to $24 \%$ in Norway).

Conclusions: International variations in ovarian cancer survival by stage exist with the largest differences observed in the oldest age group with advanced disease. This finding endorses further research investigating international differences in access to and quality of treatment, and prevalence of comorbid conditions particularly in older women with advanced disease. 
Ovarian cancer is the 8th most common cancer (excluding easily treatable, non-melanoma skin cancer) and the 5th leading cause of cancer death among women in high-income countries [1]. While incidence rates have decreased across most high-income countries over the past three decades, mortality rates declined in a slower pace [2]. Ovarian cancer symptoms are non-specific and early stage ovarian cancer is often asymptomatic. Several early detection methods have been introduced for ovarian cancer, however, currently screening is not feasible due to the low sensitivity and specificity of available tests [3]. Chemotherapy regimens for ovarian cancer have evolved with recent advances including the introduction of anti-angiogenic therapy and the use of targeted therapies such as poly(adenosine-diphosphate ribose) polymerase (PARP) inhibitors [4]. cancer survival between high-income countries $[5,6]$. There are many complex factors that impact cancer survival, including age and stage at diagnosis, as well as availability of diagnostic resources and access to optimal treatments $[5,7]$. A previous study by the International Cancer Benchmarking Partnership (ICBP) showed that more than half of all ovarian cancer cases occurred in women older than 65 years, and the majority of women were diagnosed with advanced stage disease [8]. Thus, understanding stage distribution as well as survival by stage as it relates to age at ovarian cancer diagnosis is essential to inform improvements in policy and practice. across seven high-income countries. The ICBP is a collaboration of population-based cancer registries, clinicians, researchers, and policymakers from countries with similar cancer registry coverage, national health system expenditure and universal access to healthcare. Using the most upto-date, real-world data, the ICBP investigates international differences in cancer survival in order to identify areas where practice can be improved through evidence-based recommendations. 

was previously described by Arnold and colleagues (2019) [9]. Data for ovarian cancer cases were obtained from 21 population-based cancer registries (PBCRs). These included Australia (New South Wales (NSW), Victoria, and Western Australia), Canada (Alberta, British Columbia, Manitoba, New Brunswick, Newfoundland, Nova Scotia, Ontario, Prince Edward Island, Quebec, and Saskatchewan), Denmark, Ireland, New Zealand, Norway, and the United Kingdom (UK) (England, Northern Ireland, Scotland, and Wales). We only included all cases with diagnosis of first primary ovarian cancer, including malignant cancer of the ovary (C56 - all subtypes), fallopian tubes (C57.0 - all subtypes), and peritoneum (C48.1-2 with the following histology: 8010-8035, 8041-8046, 8050- 8148, 8160$8231,8246,8250-8530,8541,8550-8576,8590-8670,8931,8933,8934,8935,8950,8959,8980-$ $8982,9000,9014,9015,9060-9090,9100,9110)$, defined according to the International Classification of Disease (ICD) $10^{\text {th }}$ revision. Cases diagnosed from 1 January 2010 to 31 December 2014 were included in the study, except for Ireland where at the time of collection data for incident cases were only available until 2013. The women were followed until 31 December 2015, except for two jurisdictions in Canada (Ontario and Newfoundland) where cases were followed until 31 December 2014. Initially, 58,161 women were included in the study (Supplementary figure S1). invasive tumors (i.e. benign, in situ or those with uncertain-malignant potential) and all tumors with specified 'borderline' histology codes- $8442,8451,8462,8472$, and 8473 - based on ICD for Oncology $3^{\text {rd }}$ revision. For survival analyses, death certificate only cases, cases identified at autopsy, as well as cases with missing month or year for the date of diagnosis or last contact were excluded. The analysis was also limited to those aged $15-99$ years at diagnosis. Additional quality controls on stage at diagnosis were carried out, including for inconsistencies in tumor behavior, and basis of diagnosis. There were 56,818 cases in the study after these exclusions. Data from cancer registries with more than $50 \%$ missing or unknown stage at diagnosis for the combined period (2010-2014) (Australia - Victoria and Western Australia; and Canada - British Columbia, New Brunswick, Ontario, and Quebec) were excluded from all analyses involving stage. 
$\underline{\text { Stage at diagnosis }}$

Each participating cancer registry were asked to provide all available data on stage at diagnosis including the Surveillance, Epidemiology and End Result (SEER) Summary Stage 2000 (SS2000), FIGO staging with sub-stage categories (e.g. 1a, 1b, 1c), as well as individualized clinical and pathological information on the extent of the tumor $(\mathrm{T})$, nodal involvement $(\mathrm{N})$, and metastasis (M). Pathological data were prioritized over clinical data for $\mathrm{T}$ and $\mathrm{N}$, while clinical $\mathrm{M}$ was prioritized over pathological M. For the diagnosis period included in the analysis, the TNM edition $\left(5^{\text {th }}, 6^{\text {th }}\right.$ or $\left.7^{\text {th }}\right)$ used varied between registries or years, however, the TNM criteria remained the same between editions for ovarian and fallopian tube cancers.

\section{Australia \& New Zealand}

Australia and New Zealand only had SEER staging data. Therefore, these countries were not included in the survival analysis by TNM or FIGO stage. Data on stage at diagnosis in Australia was only available for NSW.

\section{Canada}

Individualized TNM data were available for most Canadian provinces included in the study (Alberta, Newfoundland, Nova Scotia, Prince Edward Island, and Saskatchewan), and individualized TNM derived from Collaborative stage [10] was available for Manitoba. These provinces also provided SEER stage information.

\section{Denmark \& Ireland}

Cancer registries in Denmark and Ireland provided individualized TNM data. For both countries, TNM information was converted to SEER stage.

\section{Norway}

FIGO staging was available for almost half of the Norwegian ovarian cancer cases. To account for the remaining portion of cases, Norway has a specific coding system describing the extent of the disease based on information collected from clinical and pathological forms. This information was then converted to SEER staging and provided by the cancer registry.

\section{United Kingdom}


All four national cancer registries in the UK had data on individualized TNM, as well as FIGO staging. Additional stage information was integrated in the $\mathrm{T}, \mathrm{N}$ and $\mathrm{M}$ variables by the cancer registries in England and Wales. This registry derived T, N, and M information was used for England and Wales when converting to SEER staging, and supplemented with FIGO stage.

Mapping SEER stage

Coding and classification of stage at diagnosis varied between cancer registries.

Consequently, for comparisons, stage information from cancer registries utilizing the TNM or FIGO staging system was converted to a mapped stage with four stage categories (i.e. 'localized', 'regional', 'distant', and unknown/missing stage based on SS2000) by using an algorithm proposed by Walters and colleagues (2013), which is summarized in Supplementary table S2 [11, 12]. A validation study was performed using our current data (2010-2014) from selected Canadian provinces

(Supplementary table S3). Ovarian and fallopian tubes cancer cases with known SEER stage data and with mapped SEER stage derived from TNM data were included in the validation analysis. Mapped SEER stage was generated using the ICBP algorithm and compared to the SEER stage data originally provided by the cancer registries. The validation study using Canadian data showed that $1.9 \%$ of the 2,456 cases evaluated had mapped SEER stage that did not match the original SEER stage data provided by the cancer registry. In the study, survival analysis by stage was performed for both TNM and SEER staging.

$\underline{\text { Statistical analysis }}$

Survival by TNM or FIGO stage was calculated for Canada, Denmark, Ireland, and the UK. FIGO staging data with sub-categories was prioritized over individualized TNM clinical and pathological information for TNM stage. Nodal involvement coded as "NX" was assumed to be N0, and metastasis coded as "MX" was assumed to be M0. In the current study, stage I-IV was used to refer to TNM or FIGO stage.

Supplementary figure S4 illustrates the process to harmonize stage for ovarian cancer.

222 Australia and New Zealand only had SEER stage data, while Canada had SEER stage as well as 
individualized TNM. For cases where SEER data is missing or unknown in Canada, the individualized TNM data was used to generate mapped SEER stage. In Norway, mapped SEER stage was generated for cases with FIGO staging. SEER stage data derived from the extent of disease was then used when FIGO staging was not available. For Denmark, Ireland and UK, where SEER staging data were not available, TNM and FIGO staging were mapped to SEER. Individualized TNM data were initially used for mapping to SEER, followed by FIGO, when available. All cancer of the peritoneum was assigned 'distant' stage.

For cases with missing stage at diagnosis, stage was imputed using multinomial logistic regression with the following covariates: age (fitted with splines), vital status, and the Nelson-Aalen estimator of the cumulative hazard (survival time). Year at diagnosis treated as a factor was also added in the regression model. Additionally, the model allowed each cancer registries to have different coefficients specifically for countries with multiple registries, namely Canada and UK. Furthermore, individual regions in England were considered in the regression model when imputing stage for the UK. Thirty imputations were performed, and results from each imputation were aggregated according to Rubin's rule to estimate net survival (NS). NS is a ratio of the overall survival of individuals with ovarian cancer and the expected survival from the similar population without ovarian cancer determined using the lifetables of the general population assuming cancer death is negligible in the general population [13]. To account for population difference, the background mortality of the general population was derived from life tables from each cancer registry containing all-cause death rates by sex, age, and calendar year from 1995 to 2015 . Additionally, the $95 \%$ confidence intervals were also presented for all NS estimates.

The Pohar-Perme estimator was used to estimate the 1-year and 3-year NS using the period analysis approach for the most recent 3-year period: 2012-2014, except for Ireland (2011-2013) [14]. All cases that are alive in any point of the 3-year period contributed to the survival analysis, including cases diagnosed prior to 2012 (2011 for Ireland). All-ages and stage-specific age-standardized NS were calculated using the International Cancer Survival Standard (ICSS) weights with five age categories: $15-44,45-54,55-64,65-74$, and 75-99 years [15]. Stage categories with less than 30 cases were excluded in the stage-specific survival analysis. For cancer registry-specific survival 
analyses, registries with less than 100 cases were excluded since the estimates were unstable (i.e. Prince Edward Island, Canada). All analyses in the study were performed using Stata/IC version 14.2 (StataCorp. 2015. Stata Statistical Software: Release 14.2; College Station, TX: StataCorp LP).

\section{Results}

$\underline{\text { Patients' characteristics including cancer stage by country }}$

The median age at diagnosis ranged from 63 years in Canada to 67 years in Denmark and UK

(Table 1). The proportion of cases with unknown stage was smallest in Canada (TNM=3.6\%;

SEER $=3.0 \%)$ and largest in the UK $(\mathrm{TNM}=27.4 \%$; SEER $=27.1 \%)$. There were minimal differences in the stage distribution before and after unknown stage at diagnosis was imputed. The largest proportion of cases were diagnosed at advanced stage, and variations between countries were minor for both TNM stage, as well as, SEER stage with 'distant' stage ranging from $63.7 \%$ in Canada to $71.3 \%$ in Norway after imputing unknown stage at diagnosis. Women aged 15-44 years were predominantly diagnosed with 'localized' and 'regional' disease (Supplementary figure S5). Increasing proportions of advanced stage disease were observed with increasing age. The older age group (e.g. 75-99 years) were more commonly diagnosed with 'distant' stage ovarian cancer compared to women aged 15-44 years; i.e. $77.9-86.7 \%$ versus $29.4-45.1 \%$, respectively.

\section{$\underline{\text { All-ages and age-specific survival }}$}

Marked differences in all-ages NS were observed between countries at 1 and 3 years after diagnosis (including all cancer registries regardless of stage completeness) (Table 2 and Figure 1). For all cases combined, Ireland had the lowest 3-year NS (NS 44.8\%, 95\% CI 42.0-47.5) followed by New Zealand (NS 45.5\%, 95\% CI 42.4-48.6), while Norway ranked highest (NS 57.2\%, 95\% CI 54.659.7) followed by Australia (NS 56.4\%, 95\% CI 54.4-58.2) and Denmark (NS 53.6\%, 95\% CI 51.255.9) (Table 2B). A similar pattern was observed in 1-year NS (Table 2A). The 1-year and 3-year NS estimates using the original data with missing stage are presented in Supplementary table S6. Minimal variations in NS estimates were observed when comparing original un-imputed data versus data with imputed stage at diagnosis for missing or unknown stage (Supplementary figure S7). Inter- 
jurisdictional differences (between cancer registries within the same country) in 1-year and 3-year NS were also observed (Figure 1, Supplementary table S8-10).

Generally, survival decreased with increasing age at diagnosis and women in the oldest age group (75-99 years) had the lowest survival. Survival also varied between countries in each age category with the widest difference seen in women aged 65-74 years. The 3-year all-ages NS for this age group ranged from $40.1 \%(95 \%$ CI $34.9-45.3)$ to $60.1 \%$ (95\% CI 55.4-64.5) in Ireland and Norway, respectively. A similar pattern was observed for 1-year survival.

\section{$\underline{\text { Stage-specific survival }}$}

Figure 2 illustrates 1- and 3-year NS by country and stage with details presented in Table 2. Survival markedly decreased with advancing stage with 'distant' stage exhibiting the lowest NS for all countries. Variations in 1- and 3-year NS were observed in all three SEER stage categories. Australia and Norway had the highest 3-year NS for 'distant' stage (NS 46.9\%, 95\% CI 44.0-49.8; and NS 46.7\%, 95\% CI 43.8-49.5, respectively) (Figure 2A). The UK, Ireland and New Zealand had the lowest survival for 'distant' stage cancers (NS 33.4\%, 95\% CI 32.6-34.2; NS 32.3\%, 95\% CI 29.335.3; and NS 31.6\%, 95\% CI 28.1-35.0, respectively). For countries with TNM and FIGO staging, Denmark had the highest 3-year NS for advanced disease: stage III (NS 48.7\%, 95\% CI 44.9-52.6) and IV (NS 35.1\%, 95\% CI 31.3-38.9) (Figure 2B). Similar patterns were observed for 1-year NS with slightly less pronounced international differences.

\section{Age-specific survival by stage}

International survival differences by age groups were less stark for early stage disease. The 1and 3-year NS for 'localized' cancer among women aged 15-64 years were approximately $90 \%$ or higher for all countries in the analysis. In contrast, marked differences in survival between countries were observed among women with 'distant' stage, in particular those in the two oldest age groups (6574 and 75-99 years) (Figure 3). Among women aged 65-74 years and diagnosed with 'distant' disease, the 3-year NS ranged from $28.6 \%$ (95\% CI 23.0-34.2) in Ireland to $52.4 \%$ (95\% CI 47.2 - 
57.5) in Norway; and at 75-99 years, 3-year NS ranged from 11.5\% (95\% CI 7.4-15.5) in Ireland to 25.0\% (95\% CI 20.2-29.8) in Australia (Table 2B).

$\underline{\text { Survival differences within country by stage }}$

Inter-jurisdictional differences within countries were also observed (Supplementary table S8-10). Among Australian cancer registries, Victoria had the lowest all-ages 3-year NS of 55.8\% (95\% CI 52.9-58.6) and Western Australia had the highest (NS 60.8\%, 95\% CI 56.1-65.1). In Canada, 3-year NS ranged from 48.1\% (95\% CI 41.4-54.5) in New Brunswick to 53.3\% (95\% CI 46.7-59.4) in Manitoba. Among women with 'distant' stage, the 3-year survival ranged from 31.9\% (95\% CI 25.138.7) (Nova Scotia) to $38.6 \%$ (95\% CI 34.4-42.8) (Alberta). Within the UK, Northern Ireland (NS 42.5\%, 95\% CI 38.4-46.5) showed the lowest 3-year all-ages NS, and England exhibited the highest (NS 47.9\%, 95\% CI 47.2-48.7). For the 'distant' stage the 3-year NS ranged from $26.9 \%$ (95\% CI 22.6-31.2) (Northern Ireland) to 34.2\% (95\% CI 33.4-35.1) (England). Similar patterns were observed for 1-year NS.

\section{Discussion}

This study presents the most up-to-date estimates of all-ages, stage- and age-specific 1-year and 3-year NS for ovarian cancer across seven high-income countries. NS was highest in Norway, Australia, and Denmark followed by Canada, whereas the UK, New Zealand and Ireland exhibited lower NS. Survival differences between countries were most pronounced in older women and women with 'distant' stage disease. The latter represents the majority of ovarian cancer cases. Consequently, the largest variation in survival was observed for women with both characteristics- older age women with advanced disease. Higher NS in Norway, Australia, and Denmark specifically for advanced stage cancers and for older women contributed to their higher all-ages NS.

In our study, survival estimates for less-advanced stage ('localized' and 'regional') were dramatically higher than 'distant' stage, and younger women were commonly diagnosed with lessadvanced stage disease. Although uncommon, non-epithelial ovarian cancer are more often found in younger women and this may contribute to higher survival in less-advanced stage [16]. In contrast to 
epithelial ovarian cancer, survival in non-epithelial ovarian cancer, such as germ cell and sex cordstromal tumors, is generally higher [17].

Compared to the previous ICBP study of women diagnosed with ovarian cancer from 20042007, all-ages NS has increased in all countries with marked improvements in Denmark and Norway [8]. The centralization of healthcare services for the management of ovarian cancer has previously been associated with survival increases in several countries, and may partly explain the improved survival in some countries in the current study [18-21]. The ICBP previously reported lower ovarian cancer survival in Denmark [8]. However, the implementation of a national cancer patient pathway in Denmark has had a profound effect on reducing delays in diagnosis and treatment [21]. While some participating countries have conducted similar reforms, these changes have been more recent [22].Thus, the expansion of health systems and improvements in the organization of treatment services may potentially improve outcomes and likely narrow the differences in survival.

In our study, the majority of women with ovarian cancer are diagnosed with advanced stage.

Differences in the stage distribution between countries may indicate differences in patients' helpseeking, recognition of signs and symptoms in primary care, timely referral, as well as diagnostic practices, and may partially explain international differences in overall survival [23, 24]. More recently, positron emission tomography combined with computed tomography (PET-CT) has been increasingly used for diagnosis and staging in women with advanced ovarian cancer [25]. A Danish study reported increase in stage IV ovarian cancer over the calendar period 1995-2012, which may be related to upstaging due to PET-CT [21, 26, 27]. Tools that are able to more accurately stage ovarian cancer, such as the PET-CT, leads to patients receiving more appropriate treatments, and are thus likely to improve survival. Upstaging may partly contribute to higher survival among patients with advanced disease in some countries observed in this current study. Increased access to and use of PET-CT for ovarian cancer diagnosis may therefore improve staging and may result in further improvements in, and comparability of, survival by stage in the future.

Net survival varied substantially with age at diagnosis as women aged 75-99 years consistently had the lowest NS. The international survival gap was also large for this age group. Variation in ovarian cancer survival between younger and older patients has previously been reported 

women [29]. Other factors, such as different histology present in different age groups, differences in patient' performance status or comorbidity, delays in diagnosis, and variation in cancer treatment, might explain the survival gap between younger and older patients [30, 31]. Furthermore, older women have been shown to receive suboptimal treatment compared to younger women $[29,32]$.

Better understanding of ovarian cancer treatment practices among older women, and improvements in the way older women are clinically reviewed (e.g. performance status) and staged will lead to more appropriate treatment planning, increasing the amount of radical treatment given regardless of age. This subsequently could lead to better outcomes and reductions in international survival variation. Variation in the care and management of ovarian cancer patients, as well as adherence to treatment guidelines, may also potentially contribute to international variations in survival. The standard treatment for ovarian cancer involves cytoreductive surgery followed by chemotherapy [33]. Radical surgery to achieve 'zero residual disease' at primary cytoreduction surgery is an important determinant of survival for women with advanced disease [34]. However, some women with advanced disease may not receive cancer-directed treatment due to varying reasons including patient's choice and poor condition of the patient [35]. Overall, recommendations in clinical practice guidelines for ovarian cancer, particularly surgery, have generally remained consistent across countries $[31,36$, 37]. Within the study period (2010-2014) neoadjuvant chemotherapy (NACT) followed by interval cytoreduction surgery was increasingly utilized in the treatment of advanced ovarian cancer, however, the effect of NACT in ovarian cancer survival continuous to be debated [38-40]. Nevertheless, it is plausible that international differences in the uptake of NACT may have also contributed to the observed survival variation.

\section{Strengths and limitations}

In our current study, all datasets were subjected to extensive cleaning, harmonization and checking prior to analysis to ensure the highest possible data quality. However, completeness and accuracy of data could vary between cancer registries. In general, the availability of stage at diagnosis had improved in the last study period for all cancer registries included in the analysis. The study was 
limited to cancer registries with complete stage data for 50\% or more of the cases for 2010-2014 (i.e. excluding some cancer registries within Canada and Australia). As a result, the estimated NS presented in the current study may not be representative of the nation-wide survival, and may only be applicable for cancer registries included in the analysis. Some registries also had relatively small numbers of cases, especially in the younger age groups, resulting in unstable NS and wider confidence intervals.

Additionally, the analysis was restricted to 2010-2014 since the completeness of stage data in the years prior to this period was lower in most cancer registries. Stage at diagnosis was also imputed for cases that had missing or unknown stage. The missing cases may be missing not at random (MNAR); consequently, a sensitivity analysis was performed removing survival time variable to the regression model. The analysis showed no difference in the stage distribution and NS estimates. Moreover, staging classification varied widely across countries, and thus a mapped stage was determined for cancer registries that lack SEER stage by using a stage conversion algorithm previously developed by Walters and colleagues (2013) [12]. Based on our validation study using the Canadian data (2010-2014) misclassification of some cases has occurred in this study, although, the proportion of the cases misclassified is likely small. Consequently, this limitation should be carefully considered when making inferences of the results. NS by TNM stage was also presented in the current study for countries with only TNM and/or FIGO stage data. Similar patterns were observed in SEER and TNM stage analyses.

Differences in cancer registry practices and the quality of the stage data between cancer registries may also potentially contribute to the differences in estimates of survival by stage that were observed in the current study. It is therefore important to consider these variations when making comparisons between countries and cancer registries. The study only included long established PBCRs with high-quality data, and information from each cancer registry was carefully reviewed and processed independently. Nevertheless, stage comparisons remain challenging since the completeness of staging data, as well as the staging system used, varied between cancer registries and countries. The current study highlights the need for a common international staging system to perform more accurate cancer survival comparisons between countries. For the purpose of international 
comparisons, our main results are presented using SEER staging as well as TNM staging when available. FIGO and TNM staging is more detailed and regularly updated according to the most recent clinical evidence and should be used when available.

Conclusion

Our study highlights existing international survival differences amongst women diagnosed with ovarian cancer in seven high-income countries. The findings showed that survival differences between countries were most pronounced for older women and women with advanced disease at diagnosis. Survival variations between countries are suggestive of differences in access to and quality of care, adherence to national and international guidelines, differences in surgical philosophy and treatment approaches, prevalence of co-morbidities and the organization of healthcare services across countries - all factors which warrant further investigation and will be explored as part of the second phase of ICBP.

\section{Acknowledgements}

The ICBP is funded by the Canadian Partnership Against Cancer; Cancer Council Victoria; would also like to thank the ICBP management team of Cancer Research UK for managing the program, the ICBP SurvMark-2 Local Leads for advice to understand the data, for their contributions to the study protocol and interpretation of the results and the ICBP Clinical Committees for their advice. We are also grateful to the ICBP SurvMark-2 Academic Reference Group for providing independent peer review and advice for the study protocol and analysis plan development. Finally we are thankful to the ICBP Program Board for their oversight and direction. A complete list of all investigators can be found in page 2 of the Supplementary Materials. 
446 / World Health Organization, the authors alone are responsible for the views expressed in this article

447 and they do not necessarily represent the decisions, policy or views of the International Agency for

Research on Cancer / World Health Organization.

449

\section{Conflict of interest}

None of the authors have any potential conflicts (financial, professional, or personal) related to the manuscript to disclose.

\section{Authors' contribution}

Citadel Cabasag planned and carried out the analysis with the supervision of Isabelle

Soerjomataram, and wrote the first draft of the manuscript. Isabelle Soerjomataram, Freddie Bray, and

Melina Arnold conceived the project, participated in the study design and coordination, and provided important intellectual content. Mark Rutherford aided in the study design, the statistical analysis, and the interpretation of the results. John Butler provided critical revision and gave consultation on the figures. Aude Bardot and Jacques Ferlay contributed to data preparation and management. All other authors have reviewed the manuscript and provided critical feedback. All authors gave their approval to submit the manuscript.

\section{References}

465 [1] Global Cancer Observatory. Lyon: Intenational Agency for Research on Cancer.

466 [2] Malvezzi M, Carioli G, Rodriguez T, Negri E, La Vecchia C. Global trends and predictions in 467 ovarian cancer mortality. Ann Oncol. 2016;27:2017-25.

468 [3] Henderson JT, Webber EM, Sawaya GF. Screening for Ovarian Cancer: Updated Evidence Report and Systematic Review for the US Preventive Services Task Force. JAMA. 2018;319:595-606.

470 [4] Lopez J, Banerjee S, Kaye SB. New developments in the treatment of ovarian cancer--future perspectives. Ann Oncol. 2013;24 Suppl 10:x69-x76. 
472 [5] De Angelis R, Sant M, Coleman MP, Francisci S, Baili P, Pierannunzio D, et al. Cancer survival

473 in Europe 1999-2007 by country and age: results of EUROCARE--5-a population-based study. Lancet

474 Oncol. 2014;15:23-34.

475 [6] Allemani C, Matsuda T, Di Carlo V, Harewood R, Matz M, Niksic M, et al. Global surveillance of 476 trends in cancer survival 2000-14 (CONCORD-3): analysis of individual records for 37513025

477 patients diagnosed with one of 18 cancers from 322 population-based registries in 71 countries.

478 Lancet. 2018;391:1023-75.

479 [7] Sant M, Chirlaque Lopez MD, Agresti R, Sanchez Perez MJ, Holleczek B, Bielska-Lasota M, et 480 al. Survival of women with cancers of breast and genital organs in Europe 1999-2007: Results of the 481 EUROCARE-5 study. Eur J Cancer. 2015;51:2191-205.

482 [8] Maringe C, Walters S, Butler J, Coleman MP, Hacker N, Hanna L, et al. Stage at diagnosis and 483 ovarian cancer survival: evidence from the International Cancer Benchmarking Partnership. Gynecol Oncol. 2012;127:75-82. [9] Arnold M, Rutherford MJ, Bardot A, Ferlay J, Andersson TM, Myklebust TA, et al. Progress in cancer survival, mortality, and incidence in seven high-income countries 1995-2014 (ICBP SURVMARK-2): a population-based study. Lancet Oncol. 2019.

[10] Collaborative Stage Data Collection System. American Joint Committee on Cancer; 2019. Institute; 2001.

492 [12] Walters S, Maringe C, Butler J, Brierley JD, Rachet B, Coleman MP. Comparability of stage data in cancer registries in six countries: lessons from the International Cancer Benchmarking Partnership. Int J Cancer. 2013;132:676-85. overview of measures, uses, and interpretation. J Natl Cancer Inst Monogr. 2014;2014:145-86. [14] Perme MP, Stare J, Esteve J. On estimation in relative survival. Biometrics. 2012;68:113-20.

498 [15] Corazziari I, Quinn M, Capocaccia R. Standard cancer patient population for age standardising survival ratios. Eur J Cancer. 2004;40:2307-16. 
500

501

502

503

504

505

506

507

508

509

510

511

512

513

514

515

516

517

518

519

520

521

522

523

524

525

[16] Berek JS, Bast RC. Nonepithelial ovarian cancer. In: Kufe DW, Pollock RE, Weichselbaum RR, et al., editors. Holland-Frei Cancer Medicine. 6th ed ed. Hamilton (ON): BC Decker; 2003.

[17] Holscher G, Anthuber C, Bastert G, Burges A, Mayr D, Oberlechner E, et al. Improvement of survival in sex cord stromal tumors - an observational study with more than 25 years follow-up. Acta Obstet Gynecol Scand. 2009;88:440-8.

[18] Dahm-Kahler P, Palmqvist C, Staf C, Holmberg E, Johannesson L. Centralized primary care of advanced ovarian cancer improves complete cytoreduction and survival - A population-based cohort study. Gynecol Oncol. 2016;142:211-6.

[19] Aune G, Torp SH, Syversen U, Hagen B, Tingulstad S. Ten years' experience with centralized surgery of ovarian cancer in one health region in Norway. Int J Gynecol Cancer. 2012;22:226-31.

[20] National Framework for Gynaecological Cancer Control. Surry Hills, New South Wales: Cancer Australia; 2016.

[21] Edwards HM, Noer MC, Sperling CD, Nguyen-Nielsen M, Lundvall L, Christensen IJ, et al. Survival of ovarian cancer patients in Denmark: Results from the Danish gynaecological cancer group (DGCG) database, 1995-2012. Acta Oncol. 2016;55 Suppl 2:36-43.

[22] Canada's Health Care System. Government of Canada (Gouvernement du Canada).

[23] Forbes LJ, Simon AE, Warburton F, Boniface D, Brain KE, Dessaix A, et al. Differences in cancer awareness and beliefs between Australia, Canada, Denmark, Norway, Sweden and the UK (the International Cancer Benchmarking Partnership): do they contribute to differences in cancer survival? Br J Cancer. 2013;108:292-300.

[24] Rose PW, Rubin G, Perera-Salazar R, Almberg SS, Barisic A, Dawes M, et al. Explaining variation in cancer survival between 11 jurisdictions in the International Cancer Benchmarking Partnership: a primary care vignette survey. BMJ Open. 2015;5:e007212.

[25] Nam EJ, Yun MJ, Oh YT, Kim JW, Kim JH, Kim S, et al. Diagnosis and staging of primary ovarian cancer: correlation between PET/CT, Doppler US, and CT or MRI. Gynecol Oncol. 2010;116:389-94. 
526 [26] Risum S, Hogdall C, Loft A, Berthelsen AK, Hogdall E, Nedergaard L, et al. Does the use of

527 diagnostic PET/CT cause stage migration in patients with primary advanced ovarian cancer? Gynecol

528 Oncol. 2010;116:395-8.

529 [27] Fruscio R, Sina F, Dolci C, Signorelli M, Crivellaro C, Dell'Anna T, et al. Preoperative 18F-FDG

$530 \mathrm{PET} / \mathrm{CT}$ in the management of advanced epithelial ovarian cancer. Gynecol Oncol. 2013;131:689-93.

531 [28] Wright JD, Chen L, Tergas AI, Patankar S, Burke WM, Hou JY, et al. Trends in relative survival

532 for ovarian cancer from 1975 to 2011. Obstet Gynecol. 2015;125:1345-52.

533 [29] Fourcadier E, Tretarre B, Gras-Aygon C, Ecarnot F, Daures JP, Bessaoud F. Under-treatment of

534 elderly patients with ovarian cancer: a population based study. BMC Cancer. 2015;15:937.

535 [30] Coburn SB, Bray F, Sherman ME, Trabert B. International patterns and trends in ovarian cancer

536 incidence, overall and by histologic subtype. Int J Cancer. 2017;140:2451-60.

537 [31] Norell CH BJ, Farrell R, Altman A, Bentley J, Cohen PA et al. Exploring international variations

538 in access to optimal treatment for ovarian cancer: An International Cancer Benchmarking Partnership

539 (ICBP) study. Submitted.

540 [32] Gibson SJ, Fleming GF, Temkin SM, Chase DM. The Application and Outcome of Standard of

541 Care Treatment in Elderly Women with Ovarian Cancer: A Literature Review over the Last 10 Years.

542 Front Oncol. 2016;6:63.

543 [33] Chura C J. Ovarian Cancer. BMJ Best Practice: BMJ Publishing Group Ltd; 2017.

544 [34] Bristow RE, Tomacruz RS, Armstrong DK, Trimble EL, Montz FJ. Survival effect of maximal

545 cytoreductive surgery for advanced ovarian carcinoma during the platinum era: a meta-analysis. J Clin

546 Oncol. 2002;20:1248-59.

547 [35] Zijlstra M, Timmermans M, Fransen H, van der Aa M, Reyners A, Raijmakers N, et al.

548 Treatment patterns and associated factors in patients with advanced epithelial ovarian cancer: a

549 population-based study. Int J Gynecol Cancer. 2019;29:1032-7.

550 [36] Morgan RJ, Jr., Armstrong DK, Alvarez RD, Bakkum-Gamez JN, Behbakht K, Chen LM, et al.

551 Ovarian Cancer, Version 1.2016, NCCN Clinical Practice Guidelines in Oncology. J Natl Compr

552 Canc Netw. 2016;14:1134-63. 
553 [37] Ledermann JA, Raja FA, Fotopoulou C, Gonzalez-Martin A, Colombo N, Sessa C, et al. Newly

554 diagnosed and relapsed epithelial ovarian carcinoma: ESMO Clinical Practice Guidelines for

555 diagnosis, treatment and follow-up. Ann Oncol. 2013;24 Suppl 6:vi24-32.

556 [38] Vergote I, Trope CG, Amant F, Kristensen GB, Ehlen T, Johnson N, et al. Neoadjuvant

557 chemotherapy or primary surgery in stage IIIC or IV ovarian cancer. N Engl J Med. 2010;363:943-53.

558 [39] Chi DS, Musa F, Dao F, Zivanovic O, Sonoda Y, Leitao MM, et al. An analysis of patients with

559 bulky advanced stage ovarian, tubal, and peritoneal carcinoma treated with primary debulking surgery

560 (PDS) during an identical time period as the randomized EORTC-NCIC trial of PDS vs neoadjuvant

561 chemotherapy (NACT). Gynecol Oncol. 2012;124:10-4.

562 [40] Vergote I, Leunen K, Amant F. Primary surgery or neoadjuvant chemotherapy in ovarian cancer:

563 what is the value of comparing apples with oranges? Gynecol Oncol. 2012;124:1-2.

564

565

Table and figure legends

566 Supplemental figure 1. Study population inclusion criteria

567 Supplementary table S2. T, N \& M or FIGO stage mapped to SEER staging for ovarian cancer based 568 on ICBP algorithm developed by Walters and colleagues (2013)

569 Supplementary table S3. Comparison of SEER stage data provided by the Canadian cancer registries versus mapped SEER stage generated using ICBP algorithm developed by Walters and colleagues

571 (2013)

572 Supplementary figure S4. Flowchart on stage harmonization for ovarian cancer

573 Table 1. Total number of cases by $\mathrm{TNM}^{\mathrm{a}}$ and mapped SEER stage for women diagnosed with ovarian 574 cancer in seven countries between 2010 and 2014

575 Supplementary figure S5. Proportions of women diagnosed with ovarian cancer during 2010-2014

576 by age, mapped SEER stage and country after imputation of stage at diagnosis

577 Table 2. (A) 1-year and (B) 3-year net survival (\%) and 95\% confidence interval for women 578 diagnosed with ovarian cancer between 2010 and 2014 by age, stage, and country after imputing stage 579 at diagnosis 
580

581

582

583

584

585

586

587

588

589

590

591

592

593

594

595

596

597

598

599

600

601

602

603

604

605

606

Figure 1. Age-standardized all-ages net survival for women diagnosed with ovarian cancer between 2010 and $2014^{\mathrm{a}}$ by country and jurisdiction within countries. Abbreviations: (Australia jurisdictions) $\mathrm{NSW}=$ New South Wales, VIC $=$ Victoria, WA = Western Australia; (Canada jurisdictions) $\mathrm{AB}=$ Alberta, $\mathrm{BC}=$ British Columbia, $\mathrm{MB}=$ Manitoba, $\mathrm{NM}=$ New Brunswick, $\mathrm{NF}=$ Newfoundland $\&$ Labrador, $\mathrm{NS}=$ Nova Scotia, $\mathrm{PE}=$ Prince Edward Island, $\mathrm{ON}=$ Ontario, $\mathrm{QC}=$ Quebec, $\mathrm{SK}=$ Saskatchewan; $($ UK - United Kingdom $)$ ENG = England, NIR = North Ireland, SCO = Scotland, WAL $=$ Wales, ${ }^{a}$ Ireland (2010-2013).

Supplementary table S6. Net survival (\%) and 95\% confidence interval for women diagnosed with ovarian cancer between 2010 and 2014 by age, stage, and country prior to imputation of stage at diagnosis

Supplementary figure S7. Comparison of estimated 1-year and 3-year net survival between original data with unknown or missing stage and imputed stage data for 2010-2014 study period

Supplementary table S8. Net survival (\%) and 95\% confidence interval for Australian women diagnosed with ovarian cancer in Victoria and Western Australia between 2010 and 2014 by age and stage

Supplementary table S9. Net survival (\%) and 95\% confidence interval for Canadian women diagnosed with ovarian cancer between 2010 and 2014 by age, stage, and province after imputing stage at diagnosis

Supplementary table S10. Net survival (\%) and 95\% confidence interval for women diagnosed with ovarian cancer in United Kingdom between 2010 and 2014 by age, stage, and nation after imputing stage

Figure 2. Age-standardized 1-year and 3-year net survival and $95 \%$ confidence intervals for ovarian cancer patients diagnosed between 2010 and $2014^{\mathrm{a}}$ by mapped SEER stage (A) and TNM stage (B) after imputing stage at diagnosis. Abbreviations: pop=population, ${ }^{a}$ Ireland (2010-2013).

Figure 3. Age-specific 3-year net survival (with 95\% confidence interval) among women diagnosed with 'distant' ovarian cancer between 2010 and $2014^{\mathrm{a}}$ by country after imputation of stage at diagnosis. Abbreviations: pop=population, ${ }^{\mathrm{a}}$ Ireland (2010-2013). 